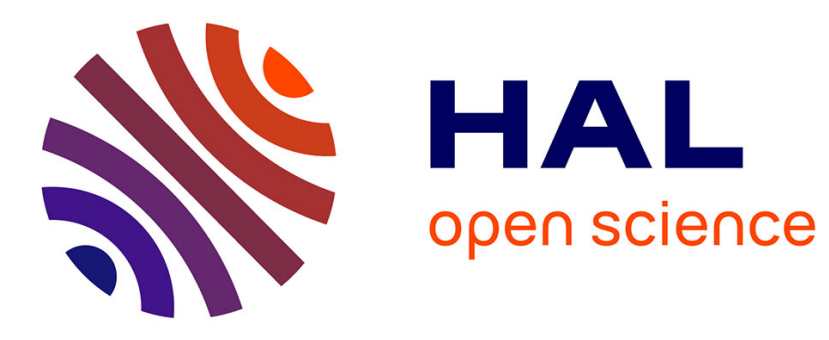

\title{
Towards Criteria Characterizing the Metrological Performance of Full-field Measurement Techniques
}

\author{
Benoît Blaysat, Jan Neggers, Michel Grediac, Frédéric Sur
}

\section{To cite this version:}

Benoît Blaysat, Jan Neggers, Michel Grediac, Frédéric Sur. Towards Criteria Characterizing the Metrological Performance of Full-field Measurement Techniques: Application to the Comparison Between Local and Global Versions of DIC. Experimental Mechanics, 2020, 60 (3), pp.393-407. 10.1007/s11340-019-00566-4 . hal-02436541

\section{HAL Id: hal-02436541 https://hal.science/hal-02436541}

Submitted on 13 Jan 2020

HAL is a multi-disciplinary open access archive for the deposit and dissemination of scientific research documents, whether they are published or not. The documents may come from teaching and research institutions in France or abroad, or from public or private research centers.
L'archive ouverte pluridisciplinaire HAL, est destinée au dépôt et à la diffusion de documents scientifiques de niveau recherche, publiés ou non, émanant des établissements d'enseignement et de recherche français ou étrangers, des laboratoires publics ou privés. 


\title{
Towards criteria characterizing the metrological performance of full-field measurement techniques
}

\section{Application to the comparison between Local and Global versions of DIC}

\author{
B. Blaysat; J. Neggers ${ }^{\dagger}$ M. Grédiac $;$ F. Sur ${ }^{\S}$
}

This is the author-manuscript version of

B. Blaysat, J. Neggers, M. Grédiac, F. Sur. Towards Criteria Characterizing the Metrological Performance of Full-field Measurement Techniques. Application to the Comparison Between Local and Global Versions of DIC. Experimental Mechanics, SEM \& Springer, 2020.

DOI: $10.1007 / \mathrm{s} 11340-019-00566-4$

\begin{abstract}
Users of full-field measurement methods like Digital Image Correlation (DIC) often aim to perform measurements with the best trade-off between spatial resolution, bias and measurement resolution. Whenever two full-field methods are compared, it is essential that these criteria are taken into consideration. Recently a metrological efficiency indicator for full-field measurements has been proposed and discussed. This indicator combines measurement resolution and spatial resolution. It has been shown to be invariant to the subset size in the case of Local DIC. The goal of this article is to discuss a method, which determines both the spatial and the measurement resolutions for a given bias for two different DIC methods, in order to obtain the metrological efficiency indicator for each of these methods. The benefit of this indicator is that it

\footnotetext{
*Université Clermont Auvergne, CNRS, SIGMA Clermont, Institut Pascal, F-63000 Clermont-Ferrand, France benoit.blaysat@uca.fr

${ }^{\dagger}$ LMT (ENS Paris-Saclay/CNRS/Université Paris-Saclay), 94235 Cachan, France

${ }^{\ddagger}$ Université Clermont Auvergne, CNRS, SIGMA Clermont, Institut Pascal, F-63000 Clermont-Ferrand, France

${ }^{\S}$ LORIA, UMR CNRS 7503 Université de Lorraine, CNRS, INRIA, Campus Scientifique, BP 239, 54506 Vandoeuvre-lès-Nancy Cedex, France
} 
does not depend on setting parameters such as the subset size, which are chosen by the user. As such, it can be considered as intrinsic to each technique, thus enabling fair comparison. Local DIC and triangular finite element based Global DIC will be the subject of this investigation. With this setting, their respective subset and triangular element sizes will be related to the spatial resolution of both methods for a given acceptable bias. By using the metrological efficiency indicator, the performance of the two methods will be compared and discussed to a new level of detail. Generally speaking, the indicator shows that the metrological performance of both methods is similar, confirming their popularity. However, it will be shown that, depending on the choice of what an acceptable bias is, one of the method may be preferred to another. The results show that for the specific DIC versions used in the study, for cases for which a significant bias is acceptable, Local DIC outperforms Global DIC, while the opposite is true in the case for which the bias requirements are more stringent. Finally, the quadratic versions of both DIC versions are shown to significantly outperform their respective linear versions.

Keywords: Digital Image Correlation, Local DIC, Global DIC, Metrological Performance, Metrological Efficiency Indicator

\section{Introduction}

Digital Image Correlation (DIC), originally developed by and for the computer vision community in the eighties [1], is now commonly used in the experimental mechanics community thanks to the seminal work of Sutton $[2,3]$. The wealth of data this technique provides is appreciated by experimentalists in order to observe mechanisms, and to calibrate their associated modelisation. However, as for any measurement tool, measurements shall come along with the metrological properties of the tools used to obtain them. This is not obvious due to the full-field measurement nature of DIC, and the scarcity of available standards. This explains why studying the metrological performance of DIC is a topical subject, e.g. $[4-15]$.

Recently, different criteria were proposed in [16] to consistently study the metrological performance of full-field measurement techniques. Moreover, a metrological efficiency indicator was introduced. It grades the overall performance of a full-field measurement technique. Firstly devoted to the Localized Spectral Analysis (LSA) approach, the introduced criterion and the associated indicator are not related to the method itself, and thus allows the comparison of the performance of several approaches although their mathematical formalisms are different. For instance, they have been used for comparison purposes between LSA and the Local version of DIC [17].

Technological developments provided the DIC community with new cameras or acquisition systems, but the DIC technique itself also evolved. For instance, from the original subset based DIC, referred to here as Local DIC, a new version that relies on the whole 
Region of Interest (RoI) has been introduced ten years ago [18-20]. This technique is referred to here as Global DIC. Global versions of DIC rely on advanced kinematics defined over the RoI of the specimen surface, contrary to conventional Local DIC, for which simpler kinematics are defined on sub-RoI called subsets. Because embedded kinematics can be chosen on purpose, Global versions of DIC perfectly correspond to the expectations of model calibration approaches. Conversely, versatility is also recommended for observation purposes, when no information about the observed material is available. In these cases, Global DIC is associated with Finite Element kinematics. Moreover, such a formalism is also well designed for calibration purposes, since the dialogue with numerical models is facilitated when the same displacement description is used for both the measurement and the simulation $[21,22]$.

In the literature, comparisons between Local and Global versions of DIC have been already carried in [23-25]. Interestingly, their conclusions are contradictory. Arguably, the reason for this difference is that the methods were not compared using consistent metrological parameters, either in terms of spatial resolution, measurement resolution or bias. The present paper relies on the recent studies dealing with the metrological performance of fullfield measurement techniques, namely LSA [26] and its comparison with Local DIC [17]. The main point of the present paper is to detail how to obtain the three quantities that contribute to the metrological performance of both the Local and the Global DIC versions, namely spatial resolution, measurement resolution and bias. The paper is organized as follows. The first section presents the criterion defining the metrological performance of any full-field measurement technique. More specifically, the metrological efficiency indicator is introduced. It scores the metrological performance of a measurement method, whatever its mathematical formalism. The second section recalls the basics of DIC. Both Local and Global versions are discussed, and tuned to enhance their metrological performance. The third section aims at comparing these two versions of DIC.

\section{Metrological performance of full-field measurement tech- niques}

\subsection{General remarks}

As for any measurement system, it is of prime importance to choose relevant parameters to describe the metrological performance of full-field measurement techniques, as well as a methodology to compare them. The main problem here is that despite recent efforts [2733], only two standards on the metrological performance of full-field measurement systems are available $[34,35]$. To the best of the authors' knowledge, although being good starting points, these standards are far from being sufficient because they do not correctly completely characterize the metrological performance of any full-field measurement techniques.

Concerning the metrological parameters, we propose to consider first the random and 
systematic errors due to image processing only. Both are thoroughly defined in Appendix A. The first one, called measurement resolution and denoted by $\sigma_{u}$, reflects the noise level in displacement maps provided by the measurement technique. This noise is due to sensor noise, which propagates up to the displacement maps. The systematic error, also called bias and denoted by $\lambda$ in the following, reflects the error with which a given full-field measurement technique systematically returns localized details in displacement maps, even in the absence of noise in the images. $\lambda$ represents a percentage decrease from the true value (that is, a DIC result of 0.45 [pixel] is $10 \%$ lower than the true value of 0.5 [pixel], so $\lambda=10 \%)$. It is clear that both $\sigma_{u}$ and $\lambda$ should ideally be as small as possible. In practice, any DIC measurement at a given pixel relies on the surrounding pixels. In what follows, let $\omega$ be the Zone of Interest $(\mathrm{ZoI})$ of a measurement. It corresponds to the support over which the calculation is performed to provide the displacement at a given pixel. Let $h$ be a length parameter characterizing it. $\omega$ and $h$ are method-dependent. $h$ corresponds, for instance, to the subset size in Local DIC. $\omega$ and $h$ are defined for Local and Global DIC in Section 3.1. Nevertheless, $\sigma_{u}$ can be adjusted with any method by enlarging more or less $\omega$, i.e. the length $h$. A consequence that every DIC user knows is that $h$ directly impacts at the same time the blur in any displacement field, in such a way that systematic error and $\sigma_{u}$ change in an opposite way as $h$ changes. In addition, for a given value of these parameters, the blur is all the higher as the details are sharp. $\lambda$ is thus related to a third quantity named spatial resolution and denoted here by $\ell_{\lambda}$. This quantity is also defined in Appendix A. It characterizes the ability of a technique to distinguish close features in a map, and this ability is all the higher as $\ell_{\lambda}$ is small. In conclusion, as summarized in Table 1 , we have at this stage three metrological parameters, namely $\sigma_{u}, \lambda$ and $\ell_{\lambda}$, which are linked for a given technique. These three parameters are influenced, for any full-field measurement technique, by a quantity set by the user which defines the calculation support, denoted here by $h$. Specifically, in this paper, $h$ will be the edge length of a subset for Local DIC, or of an equilateral triangle for Global DIC. For a given value of $h$ the spatial resolution will be different for each DIC method. The relation between $h$ and $\ell_{\lambda}$ for a given method depends on the chosen bias, the shape of the domain, the choice of matching functions and the mesh connectivity in the case of Global DIC. Determining $\ell_{\lambda}$ for a given value of $h$ and a given method is non-trivial. To ensure that all contributions are taken into account, $\ell_{\lambda}$ will be determined using a synthetic experiment, discussed in Section 2.3.

\subsection{Procedure}

In the context defined above, the first question is to know how the length $h$ of the DIC calculation is related to user-defined choices such as the subset and element sizes. The second question is how to assess the three resulting parameters $\sigma_{u}, \lambda$ and $\ell_{\lambda}$ since they can potentially all change at the same time.

A first part of the response consists in examining the product between $\sigma_{u}$ and $\ell_{\lambda}$. Indeed, $\lambda$ being assumed to be fixed by the user, $\sigma_{u}$ and $\ell_{\lambda}$ evolve in an opposite way but 
Table 1: Notation of criteria used for the assessment of the metrological performance

\begin{tabular}{l|c|c}
\hline Metric & Notation & Units \\
\hline Displacement resolution & $\sigma_{u}$ & {$[\mathrm{px}]$} \\
Bias & $\lambda$ & $\%$ \\
Spatial resolution & $\ell_{\lambda}$ & {$[\mathrm{px}]$} \\
Metrological efficiency indicator & $\alpha_{\lambda}$ & {$\left[\mathrm{px}^{2}\right]$} \\
\hline
\end{tabular}

their product is constant for $2 \mathrm{D}$ measurements,

$$
\alpha_{\lambda}=\sigma_{u} \times \ell_{\lambda}
$$

This result, rigorously demonstrated for the LSA [26] method, has also been observed in [17] to be nearly constant for Local DIC when the length $h$ changes. We will see here that the same result is obtained for Global DIC. This product $\alpha_{\lambda}$, named metrological efficiency indicator [17], is therefore intrinsic to a technique for a certain value of $\lambda$, while $h$ is extrinsic since it depends on the user.

It is proposed here to consider the following procedure in order to assess the metrological performance of Local and Global versions of DIC. The first step is to set the value of $\lambda$ a priori. For a given value of $h$, the spatial resolution is deduced by using a suitable procedure relying on synthetic data described in Section 2.3. The second step consists in determining the measurement resolution $\sigma_{u}$. Various formulas, which predict the measurement resolution when only sensor noise is considered, exist in the literature, e.g. $[4,5,7-9$, 20, 23, 36-41]. At this stage, $\ell_{\lambda}$ and $\sigma_{u}$ are known, and $\alpha_{\lambda}$ can therefore be estimated for a given value of $h$. Changing this value enables us to check that $\alpha_{\lambda}$ remains unchanged. Consequently, a calculation of $\alpha_{\lambda}$ for one value of $h$ is sufficient.

In conclusion of this section, we have here two quantities, namely $\lambda$, which is set $a$ priori by the user, and $\alpha_{\lambda}$, which is obtained through relevant calculations on synthetic images. Comparing $\alpha_{\lambda}$ obtained by each technique enables us to compare their metrological properties since the lower the value of $\alpha_{\lambda}$, the better the performance of this technique. Let us now examine in detail the synthetic images processed here to determine $\ell_{\lambda}$ and $\sigma_{u}$ for the two versions of DIC studied in this paper.

\subsection{Test case}

One pair of speckle images is obtained by considering the artificial displacement represented in Figure 1. This type of displacement field, proposed first in [42], is a sinusoidal wave of constant amplitude, but whose frequency linearly and gradually decreases from the left to the right. This enables us to analyze the spatial resolution with a single test by 
observing at which frequency the systematic errors become greater than the chosen bias. The amplitude of this wave is 0.5 pixel, so that the DIC interpolation bias is null at the points corresponding to the top and bottom of the wave. Finally, this displacement is constant along the mid-height (and axis of symmetry) of the artificial image, but the frequency of the sine wave orthogonal to this axis regularly decreases along this line. It means that plotting the cross-section of the displacement returned by DIC along this axis of symmetry is expected to show the progressive increase of amplitude of the signal returned by DIC from the left to the right. This enables us to find $\ell_{\lambda}$ for a given value of $\lambda$. The proposed methodology is first dedicated to sinusoidal displacement fields. Nevertheless, it is worth mentioning that any displacement field can be seen as the summation of sine functions, thanks to the Fourier series. With a spatial resolution $\ell_{\lambda}$, the coefficients of the term of frequencies above $1 / \ell_{\lambda}$ of the Fourrier series are impaired with, at least, a bias denoted by $\lambda$. For real applications, this definition of the spatial resolution gives thus a good estimation of the smallest detail the measurement method may confidently retrieve.

An artificial speckle was generated in both the reference and the deformed stated using the speckle generator described in [43] (see Figure 1). This pair of images, and the displacement, corresponds to the one used in the new version of the DIC Challenge [44, 45]. The main feature of this speckle generator is that it does not rely on any interpolation, which could potentially corrupt the deformed image in an uncontrolled manner.

Noise is independently added to both images. This noise is heteroscedastic to mimic at best the actual behavior of camera sensors. Its standard deviation $\sigma_{f}$ increases as the gray level $f$ increases, as modeled in [46],

$$
\forall \underline{x}_{\mathrm{p}}, \sigma_{f}\left(\underline{x}_{\mathrm{p}}\right)=\sqrt{a \times f\left(\underline{x}_{\mathrm{p}}\right)+b}
$$

where $f$ is the gray level distribution in the image, $a$ and $b$ are parameters describing the noise of a typical CCD camera. Here, $a=0.0342, b=0.2679$ have been chosen to mimic a 8 -bit camera [45].

\section{DIC}

This section briefly recalls DIC basics and formulas predicting parameters characterizing the metrological performance, when available. The practical specificities of Local and Global implementations are also discussed. The main goal of this section is to describe both implementations within the same framework.

\subsection{Mathematical formulation}

DIC aims at comparing two images of the specimen surface, i.e. the reference one $(f)$ and the current one $(g)$, in order to determine the displacement $\underline{u}$ that occurs on this specimen surface. We consider here the Region of Interest (RoI) as the part of the imaged 


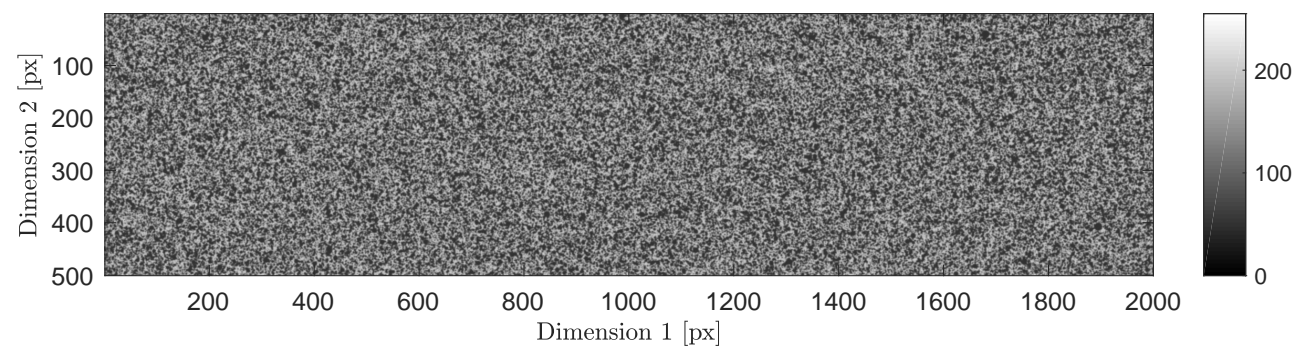

(a) Reference image

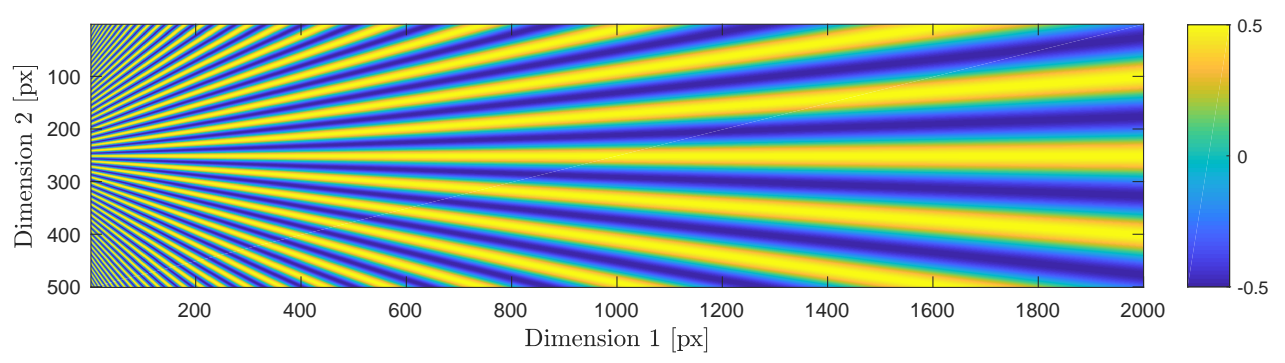

(b) Vertical component of the prescribed displacement

Figure 1: Synthetic test case, after [44, 45]: (a) reference image, generated with the tool described in [43]; (b) second component of the displacement $u_{2}$ used for computing the current image. This completely defines the artificial displacement $\underline{u}=u_{2} \times \underline{e}_{2}$. This displacement allows the assessment of the spatial resolution $\ell_{\lambda}$. The measured displacement along the middle line directly highlights the filtering effect of the measurement method with respect to the displacement spatial frequency. 
specimen surface over which the displacement is searched. The RoI is of course imaged in both images $(f)$ and $(g)$. For the sake of simplicity, the surface specimen is assumed to be planar, perfectly parallel to the camera sensor, and to deform itself with the displacement $\underline{\mathbf{u}}$ within this plane. Moreover, the light path and camera sensor are considered as perfect. Consequently, the coordinate system defined by the camera sensor is used here. The brightness conservation for all pixel positions $\underline{x}_{\mathrm{p}}$ of the RoI can be written as follows:

$$
f\left(\underline{x}_{\mathrm{p}}\right) \approx g\left(\underline{x}_{\mathrm{p}}+\underline{\mathrm{u}}\left(\underline{x}_{\mathrm{p}}\right)\right) .
$$

Since $\underline{u}$ uniquely relies on Equation (3), estimating it is an ill-posed problem. A commonly accepted regularization consists in:

- introducing an approximated kinematics space $\mathcal{U}_{\omega}$ over the $\mathrm{ZoI} \omega$, which is constituted of $N_{\omega}$ pixels. Moreover, the kinematics are assumed to be linear in both cases and $\mathcal{U}_{\omega}$ is defined with a set of $N$ matching functions $\left(\varphi_{i}\right)_{1 \leq i \leq N}$. Consequently, the approximated displacement field is completely determined over $\omega$ with $N$ Degrees of Freedom (DoFs) denoted by $\left(d_{i}\right)_{1 \leq i \leq N}$ :

$$
\forall \underline{x}_{\mathrm{p}} \in \omega, \underline{u}_{\omega}\left(\underline{x}_{\mathrm{p}}\right)=\sum_{i=1}^{N} d_{i} \underline{\varphi}_{i}\left(\underline{x}_{\mathrm{p}}\right) .
$$

- Equation (3) is reformulated as a residual whose norm is finally minimized over $\omega$ with respect to the sought displacement. This defines the optimized DoFs:

$$
\boldsymbol{d}=\underset{\boldsymbol{d}^{*} \in \Re^{N}}{\operatorname{ArgMin}}\left\{\sum_{\underline{x}_{\mathrm{p}} \in \omega}\left(f\left(\underline{x}_{\mathrm{p}}\right)-g\left(\underline{x}_{\mathrm{p}}+\sum_{i=1}^{N} d_{i}^{*} \underline{\varphi}_{i}\left(\underline{x}_{\mathrm{p}}\right)\right)\right)^{2}\right\} .
$$

A matrix notation is used here, $\boldsymbol{d}$ being a $N \times 1$ vector gathering the DoF values.

The usual Sum of Squared Difference (SSD) formalism is chosen here for the definition of the residual. The interested reader can refer to [47] for a detailed study about the definitions of the residual. Its main conclusion is that the residual elaboration might help DIC convergence but has no practical impact on its solution. The optical flow integration scheme chosen here, i.e. the backward one, is also discussed in the literature. The review proposed in [48] recalls the different numerical schemes and their consequences for DIC. Interestingly, despite showing that Inverse Compositional Gauss-Newton formulation improves the metrological performance, see [37, 49], its diffusion is nowadays limited and thus not implemented here. Moreover, such implementation will impact both Local and Global versions of DIC in the same manner.

The numerical scheme employed to solve Equation (5) is a modified Gauss-Newton scheme. More details about the modification of the scheme are given in [50, 51]. The solution is incrementally improved from an initial guess $\boldsymbol{d}^{0}$ by updating $\boldsymbol{d}$ as follows

$$
\boldsymbol{d}^{\mathrm{it}+1}=\boldsymbol{d}^{\mathrm{it}}+\boldsymbol{M}^{-1} \times \boldsymbol{L} \times \boldsymbol{r}\left(\boldsymbol{d}^{\mathrm{it}}\right),
$$


where,

- matrix $\boldsymbol{L}$ is the projection of the matching functions on the reference image gradient,

$$
\forall(i, j) \in\{1, \ldots, N\} \times\left\{1, \ldots, N_{\omega}\right\},[\boldsymbol{L}]_{i j}=\underline{\nabla} f\left(\underline{x}_{\mathrm{j}}\right) \cdot \underline{\varphi}_{i}\left(\underline{x}_{\mathrm{j}}\right)
$$

- matrix $\boldsymbol{M}$ is the DIC tangent operator,

$$
\boldsymbol{M}=\boldsymbol{L} \times \boldsymbol{L}^{T}
$$

- and vector $\boldsymbol{r}\left(\boldsymbol{d}^{\mathrm{it}}\right)$ gathers the $N_{\omega}$ values of the residual,

$$
\forall \underline{x}_{\mathrm{i}} \in \omega, \quad\left[\boldsymbol{r}\left(\boldsymbol{d}^{\mathrm{it}}\right)\right]_{i}=f\left(\underline{x}_{\mathrm{i}}\right)-g\left(\underline{x}_{\mathrm{i}}+\sum_{j=1}^{N} d_{i}^{\mathrm{it}} \underline{\varphi_{j}}\left(\underline{x}_{\mathrm{i}}\right)\right) .
$$

The measurement resolution of DIC outputs has been widely studied in the literature, e.g. [7-9, 36-38] for Local DIC versions and [20, 23, 39-41] for Global ones. More recently, $[4,5]$ revisited the usual measurement resolution formula of [20] in order to define it in a uniform framework suitable to both Local and Global versions of DIC. It also takes into account subpixel displacement and noise heteroscedasticity. Noise heteroscedasticity can be taken into account by using two approaches: (i) a updated formula is proposed, in which pixelwise properties of the noise are considered and (ii) image noise is normalized by using the generalized Anscombe transform. Moreover, the validation of the proposed prediction formula is performed with experimental data. We consider here that noise heteroscedasticity impacts both versions of DIC in the same manner. This impact is overestimated using the upper bound of noise standard deviation $\sigma_{f}^{\max }$ and considering that we have the same standard deviation of the noise at any pixel. With this assumption, the upper bound of the measurement resolution associated with the DIC DoFs corresponds to the original formula of [20]. It is equal to

$$
\sigma_{d_{i}}=\sigma_{f}^{\max } \times \sqrt{2 \times\left[\boldsymbol{M}^{-1}\right]_{i i}}
$$

It should be emphasized that the above developments are sufficiently general to include many of the most popular Local and Global frameworks. Then, the differences between these two versions of DIC are detailed. More specifically, their tuning for optimizing their metrological performance is discussed.

\subsection{Local version}

The Local version of DIC $[2,3]$ relies on a local definition of the domain $\omega$, called subset, associated with a relatively low degree kinematic definition, called matching functions. Although all DoFs of the matching functions are optimized, typically, only the zero degree 
DoF, i.e. the displacements at the centers of the subsets, are kept. In the literature, investigations for optimal subset definition, e.g. [52], or for the optimizations of the kinematics in the case of fracture cases, e.g. [53], are available. Recently, a deconvolution procedure has been introduced [54]. This post-processing considerably increases the overall metrological performances of DIC outputs. However, the diffusion of such approaches is nowadays limited, so the formalisms chosen here focus on the most popular ones.

DIC calculations are performed for a multiple of squared subsets $\left(\omega_{i}\right)$ in order to cover the whole RoI. The RoI is generally covered with partially overlapping subsets and the displacements at the subset centers are then used to interpolate the displacement field for all pixels in the RoI. For this article, interpolation is avoided by applying a dense subset grid with one subset around every pixel in the RoI. Arguably, this is not common for Local DIC codes. However, it is almost always possible to configure Local DIC software to behave in this manner. It improves the accuracy of the results by removing any error due to subset-to-subset interpolation at the cost of amplifying the required computational effort.

For each pixel $\underline{x}_{\mathrm{p}}$ of the RoI, one has

$$
\left\{\begin{array}{l}
\omega_{\underline{x}_{\mathrm{p}}}^{\mathrm{L}}=\left\{\text { integer square of size } h \text { centered at pixel } \underline{x}_{\mathrm{p}}\right\} \\
\mathcal{U}_{\omega_{\underline{x}_{\mathrm{p}}}}^{\mathrm{L}}=\Re_{p}[X, Y]
\end{array},\right.
$$

where $h$ is the subset width and $\Re_{\uparrow}[X, Y]$ corresponds to the space of the two-variable polynomial functions of degree $p$. Commercial codes often rely on matching functions of the first-degree, i.e. $\uparrow=1$. However, second-degree matching functions (i.e. $p=2$ ) are also included in the discussion of this paper since it is known in the literature that they significantly enhance the metrological performance [10]. The dimensionless matching functions are defined as follows,

$$
\begin{aligned}
& \text { First-degree } \\
& p=1 \\
& \text { Second-degree } \\
& p=2 \\
& \left\{\begin{array}{l}
\underline{\varphi}_{1}^{\mathrm{L}}(\underline{x})=\underline{e}_{1} \\
\underline{\varphi}_{2}^{\mathrm{L}}(\underline{x})=\underline{e}_{2} \\
\underline{\varphi}_{3}^{\mathrm{L}}(\underline{x})=x_{1} \times \underline{e}_{1} \\
\underline{\varphi}_{4}^{\mathrm{L}}(\underline{x})=x_{2} \times \underline{e}_{1} \\
\underline{\varphi}_{5}^{\mathrm{L}}(\underline{x})=x_{1} \times \underline{e}_{2} \\
\underline{\varphi}_{6}^{\mathrm{L}}(\underline{x})=x_{2} \times \underline{e}_{2}
\end{array}\right. \\
& \left(\underline{\varphi}_{i}^{\mathrm{L}}\right)_{1 \leq i \leq 6} \text { identical to the first-degree case, } \\
& \bar{c}^{2} \underline{\underline{L}}(\underline{x})=\frac{1}{2} \times x_{1}^{2} \times \underline{e}_{1} \\
& \underline{\varphi}_{8}^{\mathrm{L}}(\underline{x})=x_{1} \times x_{2} \times \underline{e}_{1} \\
& \underline{\varphi}_{9}^{\mathrm{L}}(\underline{x})=\frac{1}{2} \times x_{2}^{2} \times \underline{e}_{1} \\
& \underline{\varphi}_{10}^{\mathrm{L}}(\underline{x})=\frac{1}{2} \times x_{1}^{2} \times \underline{e}_{2} \\
& \begin{array}{l}
\underline{\varphi}_{11}^{\mathrm{L}}(\underline{x})=x_{1} \times x_{2} \times \underline{e}_{2} \\
\underline{\varphi}_{12}^{\mathrm{L}}(\underline{x})=\frac{1}{2} \times x_{2}^{2} \times \underline{e}_{2}
\end{array}
\end{aligned}
$$

They are therefore centered on each subset, i.e. for each pixel $\underline{x}_{\mathrm{p}}$,

$$
\mathcal{U}_{\omega_{\underline{x}_{\mathrm{p}}}^{\mathrm{L}}}^{\mathrm{L}}=\operatorname{span}\left\{\left(\underline{\varphi}_{i}^{\mathrm{L}} \circ \underline{m}_{\underline{x}_{\mathrm{p}}}\right)_{1 \leq i \leq 6 \times p}\right\} \text {, with } \forall\left(\underline{x}, \underline{x}_{\mathrm{p}}\right) \in \Re^{2}, \underline{m}_{\underline{x}_{\mathrm{p}}}(\underline{x})=\frac{1}{h}\left(\underline{x}-\underline{x}_{\mathrm{p}}\right) .
$$


Local operators $\boldsymbol{M}_{\underline{x}_{\mathrm{p}}}^{\mathrm{L}}$ and $\boldsymbol{L}_{\underline{x}_{\mathrm{p}}}^{\mathrm{L}}$ of the DIC resolution scheme are directly derived from the set of functions defined above, and from Equations (8) and (9).

The dimensionless definition of the matching functions facilitates the definition of the displacement as well as the measurement resolution at each pixel. For any degree of the matching functions, the displacement at the subset center $\underline{x}_{\mathrm{p}}$ is given by the first two optimized DoF values. After Equation (10), the measurement resolution can be written as follows

$$
\forall k \in\{1,2\}, \forall \underline{x}_{\mathrm{p}} \in \mathrm{RoI}, \sigma_{u_{k}^{\mathrm{L}}}\left(\underline{x}_{\mathrm{p}}\right)=\sigma_{f}^{\max } \times \sqrt{2 \times\left[\left(\boldsymbol{M}_{\underline{x}_{\mathrm{p}}^{\mathrm{L}}}^{\mathrm{L}}\right)^{-1}\right]_{k k}} .
$$

Finally, under the assumption that the minimization perfectly converges towards the true displacement, DIC might be considered as the projector of this displacement to the space of polynomial functions [10]. In other words, DIC acts as a Savizky-Golay filter. The validation of this filtering effect of the Local DIC is performed with synthetic data in [17]. We consider in the latter the empirical definition also used in [17] and recalled in Section 2 for the spatial resolution.

\subsection{Global version}

Contrary to the Local version of DIC, the Global version relies on advanced kinematics defined over the entire RoI. First Global DIC implementations relied on FE shape functions, see [20] and more recently e.g. [25, 55]. These general kinematics are well suited when DIC is used for observation purposes. Polynomial functions [56] and more recently spline functions $[57,58]$ have also been implemented with the same goal. Moreover, the global framework of Global DIC make possible the tuning of its kinematics to match expected physical phenomena. This is performed using analytical closed-form solutions such as for fracture mechanics, e.g. [59-61], or using a Finite Element (FE) simulation of the observed phenomena, e.g. [62-65]. Finally, the FE kinematic definition also enables local enrichments, as in the case of fracture mechanics [40] or regularization corresponding to the observed phenomenon [66].

This article focuses on a general Global version of DIC. This is the regular FE-based version, which uses linear (3-noded) or quadratic (6-noded) triangular elements.

Let $\left(\underline{\varphi}_{i}^{\mathrm{G}}\right)_{1 \leq i \leq N}$ be the FE shape functions used to define the kinematics space required for solving the DIC problem defined by Equation $(5) .\left(\omega^{\mathrm{G}}, \mathcal{U}_{\omega}^{G}\right)$ is thus defined as follows

$$
\left\{\begin{array}{l}
\omega^{G}=\operatorname{RoI} \\
\mathcal{U}_{\omega}^{G}=\mathcal{U}_{\mathrm{RoI}}^{\mathrm{G}}=\operatorname{span}\left\{\left(\underline{\varphi}_{i}^{\mathrm{G}}\right)_{1 \leq i \leq N}\right\} .
\end{array}\right.
$$

For linear triangles, the FE shape functions are directly the barycentric coordinates $\boldsymbol{\eta}$,

$$
\forall i \in\{1,2,3\} \text { and } k \in\{1,2\}, \quad \underline{\varphi}_{2(i-1)+k}^{\mathrm{G}}(\underline{x})=\varphi_{i}^{\mathrm{G}}(\underline{x}) \underline{e}_{k} \quad \text { and } \quad \varphi_{i}^{\mathrm{G}}(\underline{x})=\eta_{i}(\underline{x}) .
$$


For quadratic triangles with the edge nodes located exactly at the center of the triangle vertices, the quadratic shape functions read as follows

$$
\begin{array}{ll}
\varphi_{1}^{\mathrm{G}}(\underline{x})=\eta_{1}(\underline{x})\left(2 \eta_{1}(\underline{x})-1\right), & \varphi_{4}^{\mathrm{G}}(\underline{x})=4 \eta_{1}(\underline{x}) \eta_{2}(\underline{x}), \\
\varphi_{2}^{\mathrm{G}}(\underline{x})=\eta_{2}(\underline{x})\left(2 \eta_{2}(\underline{x})-1\right), & \varphi_{5}^{\mathrm{G}}(\underline{x})=4 \eta_{2}(\underline{x}) \eta_{3}(\underline{x}), \\
\varphi_{3}^{\mathrm{G}}(\underline{x})=\eta_{3}(\underline{x})\left(2 \eta_{3}(\underline{x})-1\right), & \varphi_{6}^{\mathrm{G}}(\underline{x})=4 \eta_{3}(\underline{x}) \eta_{1}(\underline{x}) .
\end{array}
$$

The DIC operators $\boldsymbol{M}^{\mathrm{G}}$ and $\boldsymbol{L}^{\mathrm{G}}$ associated to the Global version are elaborated from the shape functions given by Equations (16) and (17), which are plugged into Equations (8) and (9).

With this formulation, DoFs $\boldsymbol{d}^{\mathrm{G}}$ correspond to the collection of the nodal displacements. Contrary to Local DIC, the interpolation of the nodal displacement field to all the pixels of the RoI is naturally defined by the shape functions, and there is no need for introducing an arbitrary pixel interpolation. Consequently, the associated measurement resolution is also connected to the shape functions. Let $\left(\boldsymbol{\varphi}_{\underline{e}_{k}}^{G}\right)_{k=\{1,2\}}$ be the two $N_{\text {RoI }} \times N$ matrices that gather the pixel values of each shape function such that:

$$
\forall k=\{1,2\}, \forall(i, j) \in\left\{1, \ldots, N_{\mathrm{RoI}}\right\} \times\{1, \ldots, N\}, \quad\left[\underline{\varphi}_{\underline{e}_{k}}^{\mathrm{G}}\right]_{i j}=\underline{\varphi}_{j}^{\mathrm{G}}\left(\underline{x}_{\mathrm{i}}\right) \cdot \underline{e}_{k} .
$$

At convergence, the displacement writes as follows

$$
\forall \underline{x}_{\mathrm{i}} \in \operatorname{RoI}, \underline{u}_{\mathrm{RoI}}\left(\underline{x}_{\mathrm{i}}\right)=\left[\underline{\varphi}_{\underline{e}_{1}}^{\mathrm{G}} \times \boldsymbol{d}^{\mathrm{G}}\right]_{i} \underline{e}_{1}+\left[\underline{\varphi}_{\underline{e}_{2}}^{\mathrm{G}} \times \boldsymbol{d}^{\mathrm{G}}\right]_{i} \underline{e}_{2},
$$

and the corresponding measurement resolution has the following upper bound [4, 23]

$$
\forall k \in\{1,2\}, \forall \underline{x}_{i} \in \operatorname{RoI}, \sigma_{u_{k}^{\mathrm{G}}}\left(\underline{x}_{i}\right)=\sigma_{f}^{\max } \times \sqrt{2 \times\left[\underline{\varphi}_{\underline{e}_{k}}^{\mathrm{G}} \times\left(\boldsymbol{M}^{\mathrm{G}}\right)^{-1} \times\left(\boldsymbol{\varphi}_{\underline{e}_{k}}^{\mathrm{G}}\right)^{T}\right]_{i i}} .
$$

No closed-form expression exists for the estimation of the bias. Consequently, the empirical estimation recalled in Section 2 will be applied.

Considering the test case defined in Section 2.3, the natural solution would be to generate a mesh with small elements on the left and large elements on the right to optimally capture the kinematics in this case. In Global DIC, such mesh adaptations are generally part of a learning process, starting with a regular mesh and iteratively improving it while observing the residual fields. Moreover, adaptive approaches are available in the literature $[25,58,67]$. They propose to increase the kinematic degree of FE shape functions or spline functions in order to optimize the kinematics description according to detailed criteria. Nevertheless, for this article, structured hexagonal grid meshes will be applied. The linear or quadratic elements are all equilateral triangles, with an edge length $h$. This is done in order to analyze for the spatial resolution analyzes of the method.

For both Local and Global DIC versions, the metrological performance is not constant over the subset or element. For the Local method, this complexity is alleviated by considering only the displacement obtained at the center of the subset. Indeed this is the point 
where the results feature the best measurement resolution is obtained [23]. However, for global methods, the matching functions define the displacement everywhere in the domain. Global DIC output maps are thus more consistent with the original cost function defined in Equation (5). A consequence of this choice is that the global error, i.e. the sum of the systematic and random errors, is minimized. However, it also means that the mapping of this error is not constant, and that it is the highest at the nodes and the lowest at the center of the elements $[4,23]$.

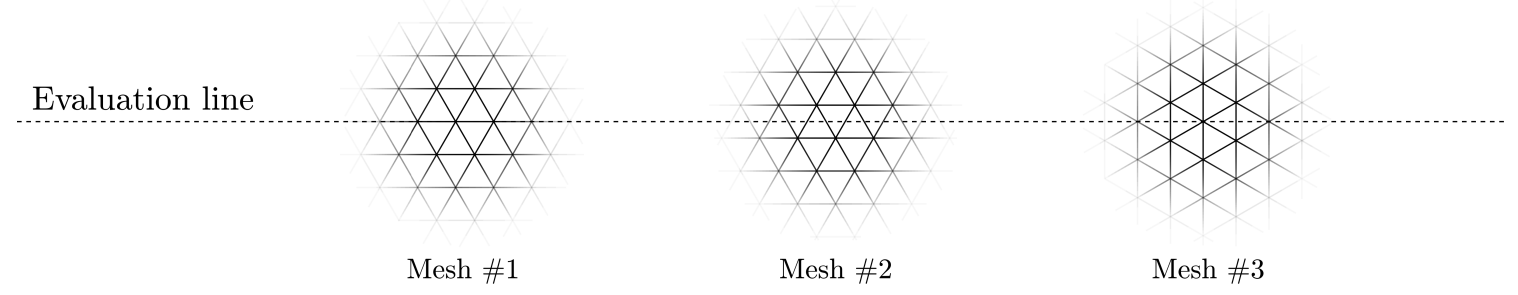

Figure 2: The mesh configurations that were analyzed. Here only a small section of the mesh is drawn. The evaluation line is superimposed at the center of the test case.

Moreover, the metrological performance of the methods will be mostly evaluated along the (horizontal) symmetric line, at the center of the test case. Since the positioning of the nodes matters, three structured meshes are introduced, each with their nodes placed differently with respect to this "evaluation line", see Figure 2. Mesh \#1 has a row of nodes exactly on the evaluation line, Mesh \#2 is positioned with the evaluation line centered between two lines of nodes, while Mesh \#3 has the pattern rotated by $90^{\circ}$ with respect to the preceding cases. It consequently has intermittently a node and an edge along the evaluation line. The goal here is not to be exhaustive but instead to test three basic mesh configurations, and use the one for which Global DIC behaves similarly than Local DIC.

\subsection{Measuring the spatial resolution $\ell_{\lambda}$}

A necessary step in the analysis of both methods is to estimate their spatial resolution. The applied test case is designed to facilitate this. The decreasing frequency of the vertical displacement field will naturally test the capabilities of the kinematic descriptions to correctly describe the test displacement field. For a given element/subset size, there will be a portion on the left-hand side of the displacement field which will be poorly described, while a portion on the right-hand side will be correctly described. The method used in this article is to choose a threshold value of the displacement accuracy, and define the wavelength at which this threshold is reached as the spatial resolution. This methodology reflects the lowpass filtering effect of the measurement method. For Local DIC, this method has been proven highly suitable. However, Global DIC is a bit more challenging 

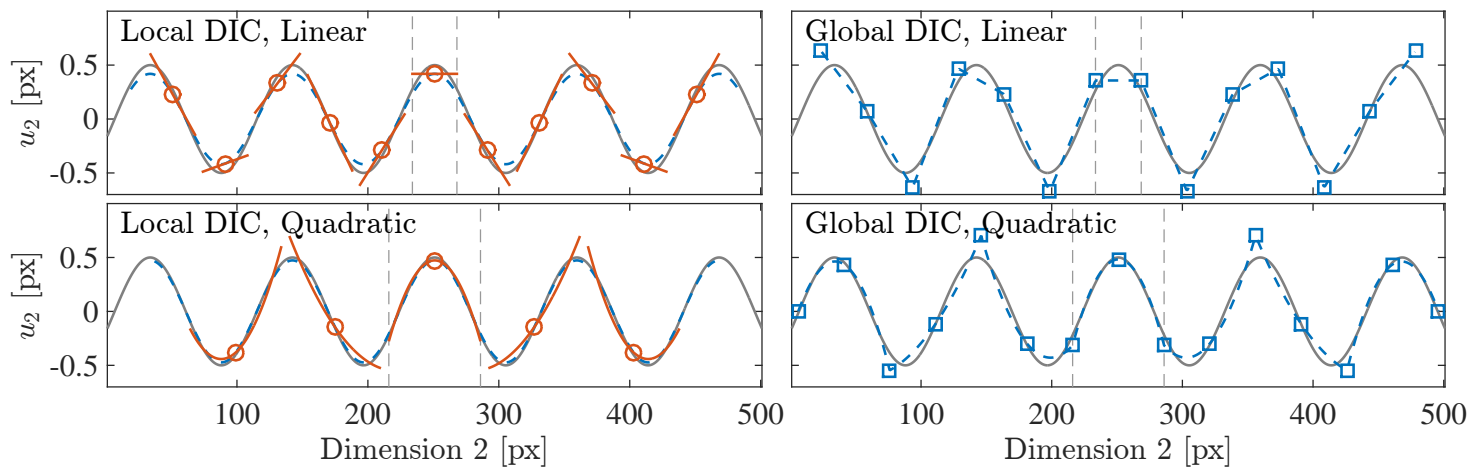

Figure 3: Visualization of the ability of the matching functions to follow a sinusoidal function. For Local DIC, only a few subsets are drawn, the finally obtained best fit is the dashed (blue) line. For Global DIC, the obtained best fit is the mesh also shown by the dashed (blue) line.

to cast in this framework.

Figure 3 schematically illustrates this issue. Local DIC subsets act like low pass filters and thus will always underestimate the maxima. Indeed, as shown in Figure 3, although the polynomial fit easily overestimated the maxima within a subset, only the conservative part of the displacement, i.e. its value at the center of the subset, is kept. The result is an additional smoothing effect, at the cost of introducing a bias. In Global DIC, the entire support of the kinematic basis is used instead of only the centers of the subsets. Consequently, the resulting displacement fields can either under- or overestimate the local curvature of the sought fields. As a consequence, no bias is induced by these fluctuations since the whole displacement field is considered to assess it. The price is to locally introduce sharp features in the error, at the node locations. The fact that there is no bias in the formulation does not mean there will be no bias. The observed errors are systematic and thus a bias is introduced. However, this bias is strongly a consequence of the interplay between the mesh and the underlying kinematics.

Figure 4a shows the obtained vertical displacement fields for Local and Global DIC for the three mesh types, and for one element/subset size, namely $h=21$ [px], using both linear and quadratic matching functions for both methods. Roughly speaking, each of the presented fields can be cut into three zones from left to right.

- Zone A) The matching functions are not able to follow the high frequencies present in this zone. The obtained displacements are unreliable.

- Zone B) The matching functions have difficulties to describe this intermediate band of frequencies. The obtained displacement fields resemble the real displacements but show severe deviations. In the case of Local DIC the deviations are in the form of an 
amplitude reduction. For Global DIC the deviations are the consequence of interplays with the structure of the mesh and the structure of the actual displacement field.

- Zone C) The matching functions are adequately able to follow these lower frequencies.

The difference between linear and quadratic matching functions is clearly emphasized by the reduction of Zones $\mathrm{A}$ and $\mathrm{B}$ towards the high frequencies for the right-hand side column of sub-figures compared to the left-hand side one. This improvement is almost entirely due to the improved spatial resolution of the quadratic matching functions given at equal element/subset size. However, the additional freedom also leads to an increased sensitivity to sensor noise. The net advantage of the quadratic functions over the linear ones will be discussed in Section 4.3 on using the metrological efficiency indicator.

The locations of the borders between the zones is abstract in this section but will be evaluated quantitatively later on. However, they present an opportunity to discuss the concept of the chosen bias $\lambda$. The spatial resolution of a method depends on how much a loss of signal amplitude is acceptable to still reconstruct the displacement for a specific frequency. Thereby, increasing $\lambda$ will move the border between Zone B and Zone $\mathrm{C}$ to the left. Thus, increasing the spatial resolution is possible at the price of accepting more artifacts. The specific value of $\lambda$ that is acceptable depends on the case. To fairly compare two methods it suffices to compare them at the same value of $\lambda$. However, it should be realized that low values of $\lambda$ will put more emphasis on the measurement resolution and high values of $\lambda$ will put more emphasis on spatial resolution and bias.

Figure $4 \mathrm{c}$ shows the vertical displacements along the evaluation line for both methods for a range of element/subset sizes. Elements and subsets are illustrated in Figure 4b. For Local DIC, the obtained displacement maxima are systematically underestimated, ranging from insignificant at low frequencies (right-hand side) to severe at high frequencies (lefthand side). This bias is reduced when reducing the subset size at the price of obtaining noisier displacement fields. This figure emphasizes the reduction in bias when going from linear to quadratic matching functions at the cost of amplifying noise. For Global DIC, the overall results are similar. Namely, for large element sizes, the error is dominated by the limited kinematic freedom while for small elements, the error is dominated by the propagation of image noise up to the displacement field. However, in contrast to the Local DIC result, the three meshes show dramatically different behavior. Here, Mesh \#2 shows similar behavior to the local DIC result. However, the displacement amplitude of Mesh \#1 increases with the wavelength, but then exceeds before ultimately approaching the correct value from the top. Lastly, Mesh \#3 intermittently under- and overestimates the displacement amplitude. It should be emphasized that this test case is exactly chosen to analyze the limits of the kinematic basis, and thus amplifies systematic errors that should not be as severe in real applications of DIC. The behavior of Global DIC is due to the interplay between the element edges and the underlying displacement fields. Deciding which mesh performs at best is arbitrary and case-dependent. The overestimation for Meshes \#1\&3 is troublesome as it complicates the definition of the chosen bias. Therefore, 


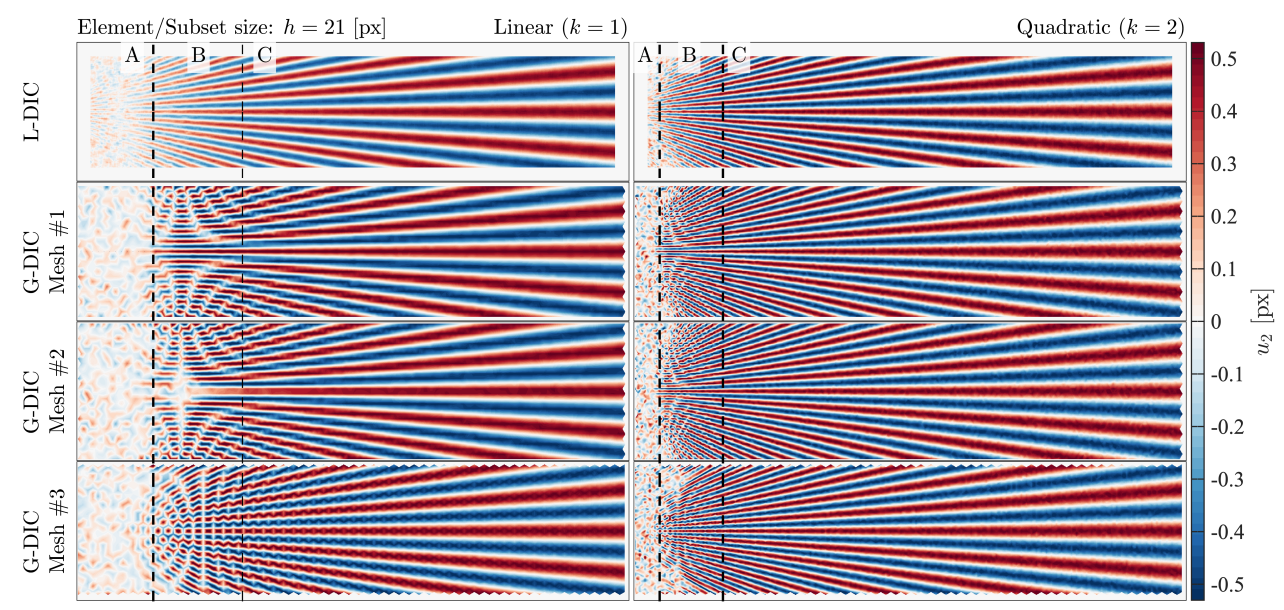

(a)

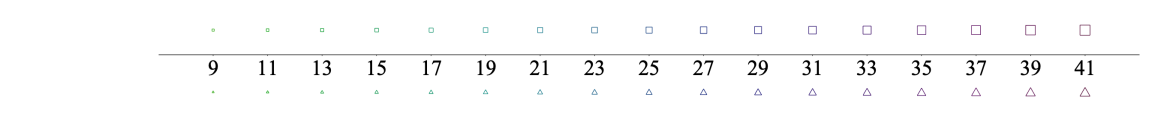

(b)

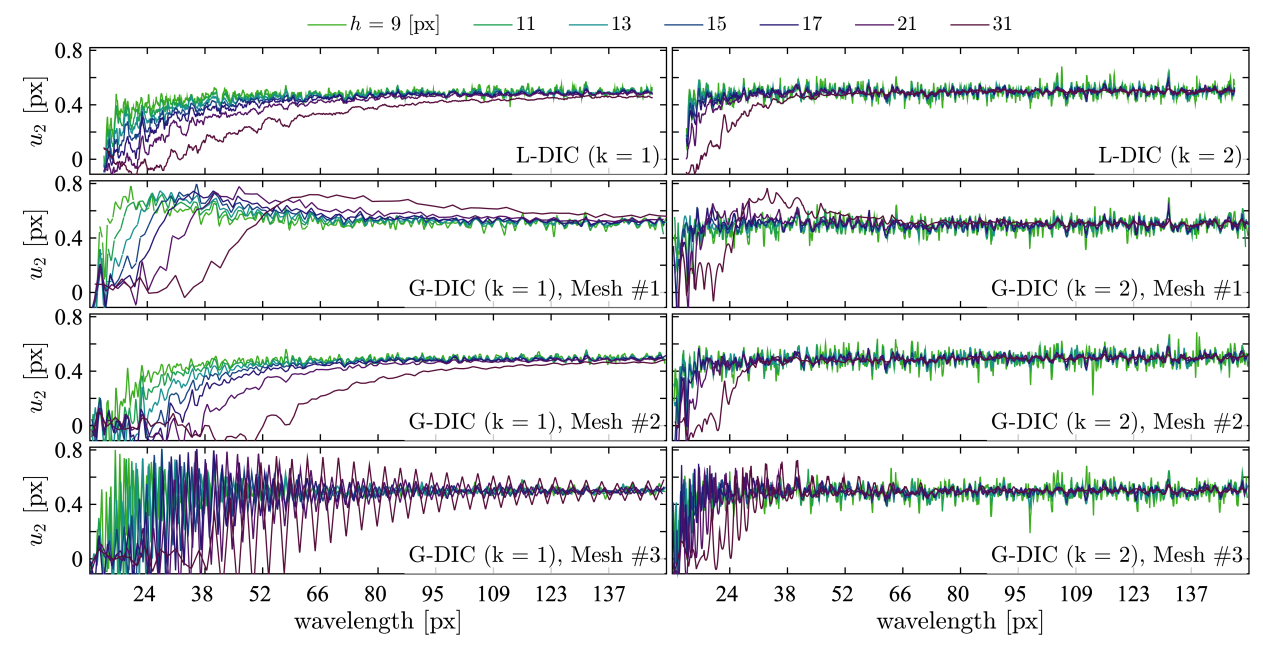

(c)

Figure 4: (a) the $u_{2}$ displacement fields as obtained for Local DIC (top row) using a subset of size 21 [px], and for Global DIC (rows 2 to 4) using an element length of 21 [px], for all three meshes for both linear (left-hand side) and quadratic (right-hand side) matching functions; (b) illustration of the elements/subsets chosen for the study. The scale used here is the same as in Figure (a); (c) the $u_{2}$ displacements along the evaluation line, as obtained by Local DIC (top row) and for Global DIC (rows 2 to 4) with both linear (left-hand side) and quadratic (right-hand side) matching functions. The subset size and element length are mentioned on the top. 
it was chosen to consider the results of Mesh \#2 in the following sections, since its behavior is most comparable to the behavior of the Local DIC.

\section{Results and discussion}

We propose here to focus first on the two parameters characterizing the metrological performance of Global and Local DIC, i.e. the measurement resolution and the spatial resolution for a bias of $\lambda=10 \%$. Then, the overall performances of both methods are compared for a range of biases thanks to the use of the metrological performance efficiency indicator.

\subsection{Spatial resolution $\ell_{\lambda}$}

The spatial resolution is empirically calculated using the displacement maps obtained by DIC applied on the synthetic data described in Section 2.3. For this purpose, we consider the middle line. The true displacement equals here exactly 0.5 [px] The relative bias associated with the measurements can thus be easily computed. Figure 5a illustrates the method used for the calculation of the spatial resolution:

- The raw DIC output is filtered using a moving average filter of width 100 [px], for smoothing local effects of image noise. As a remark, the period change of the ground truth displacement is about $5 \%$ within a window of width 100 [px].

- The intersection between the horizontal line of desired bias gives the minimum wavelength that the method can retrieve with this bias. This directly defines the spatial resolution. This calculation is performed here for a bias of $10 \%$. The choice of $10 \%$ bias ensures that all approaches correctly behave, or, in other words, that the border between Zones B and C discussed in Section 3.4 is located in a region where the interplay between DIC kinematics and ground truth displacement is reduced.

This method is applied to Global and Local DIC measurements. The obtained results are given in Figure 5b. Several remarks can be drawn from these results:

- First, for both DIC versions as implemented in this paper, the spatial resolution for elements and subsets of equal length/size is very similar. This is surprising since the edge length of a triangle element has little relation with the edge length of a square subset. It means that the connectivity of the triangles generates a support similar to the support of a square subset.

- Second, as demonstrated in [10], second-degree matching functions show better performance than first-degree ones. Considering the same element or subset size, the spatial resolution of first-degree approaches is about three times the one of the seconddegree matching functions. 


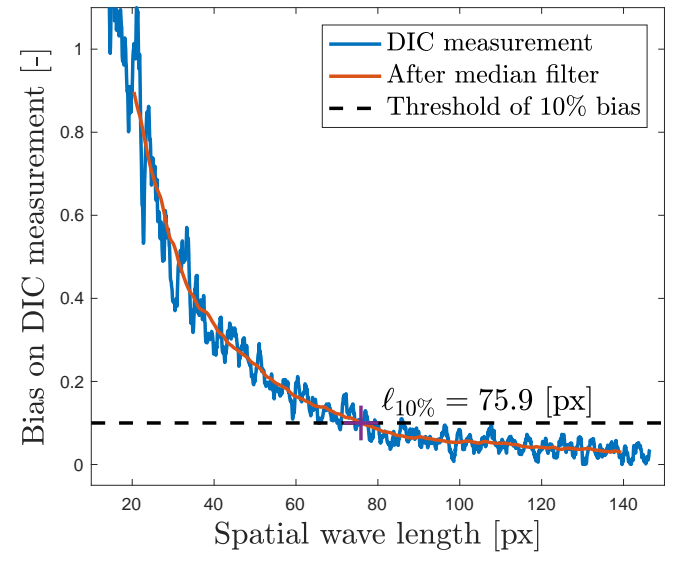

(a)

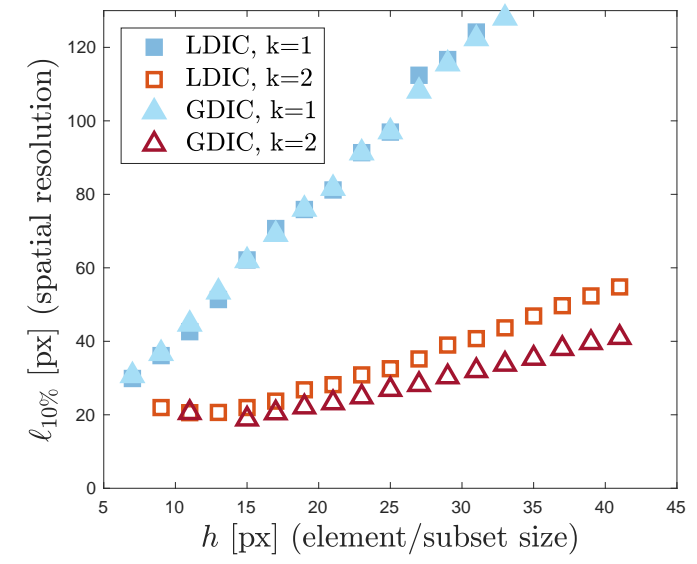

(b)

Figure 5: Spatial resolution computation and results. (a) Computation for the Local DIC case, in the case of a subset width of 19 [px]: (i) the relative bias is computed along the middle line, where the true displacement is equal to 0.5 [px], (ii) the raw DIC bias is smoothened with a median filter and (iii) the smallest wavelength with a bias equal to $10 \%$ corresponds to the spatial resolution. (b) spatial resolution as a function of the element/subset size, for first and second-degree matching functions. LDIC and GDIC respectively refer to the Local and Global versions of DIC, $k=\{1,2\}$ corresponds to the degree of the matching functions. 
- Finally, the spatial resolution appears to linearly increase with respect to the support width $h$ used for DIC calculations. This property is also demonstrated for another full-field measurement technique that is the Localized Spectrum Analysis [68]. The quadratic results start deviating from this linear behavior for $h<19$ [px]. It is assumed this is because the spatial resolution reaches its limit with this kind of speckle.

\subsection{Measurement resolution $\sigma_{u}$}

The measurement resolution is directly derived from the formulas available in the literature $[4,23]$. Employing them avoids performing numerous DIC calculations with different copies of the same noise to assess the measurement resolution. They are recalled here in Equation (14) for the Local version of DIC and Equation (20) for the Global version. These formulas allow the calculation of the standard deviation of the obtained displacement maps when affected by camera sensor noise. The standard deviations of the displacement noise are calculated for each pixel of the RoI. Nevertheless, the framework of Global DIC impacts measurement resolution in a non-homogeneous manner. Pixel gray levels are indeed weighted by their distance to the mesh nodes. Camera sensor noise does not propagate in a similar manner on the kinematics for pixels that are localized in boundary elements, or even within a single element, $c f$. $[4,23]$. To avoid such localization effects, the square root of the average variance is considered over a central area $\Omega$, defined as the middle horizontal band of 201 pixels width. Finally, only the second component of the displacement is considered, i.e. $\sigma_{u}=\left\langle\sigma_{u_{2}}\right\rangle_{\Omega}$, where $\langle\cdot\rangle_{\Omega}$ is the averaging operator over $\Omega$. The same quantity is computed for Local DIC. This measurement resolution indicator is given in Figure 6 as a function of element and subset sizes. Several remarks can be drawn from these results:

- First, and as expected, the support width $h$ drives the measurement resolution: for both DIC versions, the smaller the support width, the higher the measurement resolution. The curves follow power laws, as emphasized in Figure 6. Moreover, the first and second degree kinematics feature the same slope.

- Second, for fixed support width $h$, low degree kinematics feature better measurement resolution. Indeed, as stated in the literature, DIC methods relying on high degree matching functions are more sensitive to noise.

- Finally, the measurement resolution of both the Local and the Global versions of DIC is similar. Indeed, for equivalent support width $h$, i.e. element or subset sizes, these Global and Local versions feature the same measurement resolution.

\subsection{Metrological efficiency indicator $\alpha_{\lambda}$}

Figures 7a represents the measurement resolution as a function of the spatial resolution. This plot illustrates the compromise between spatial and measurement resolutions: an 


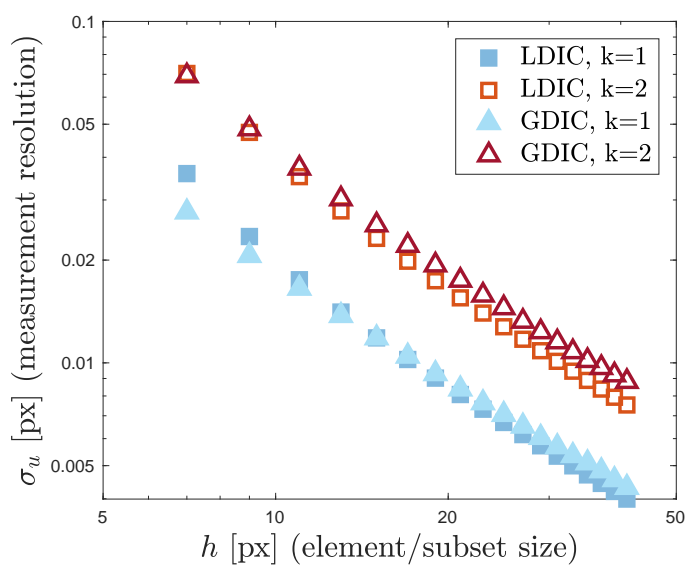

Figure 6: Measurement resolution as a function of the element/subset size. LDIC and GDIC respectively refer to the Local and Global version of DIC and $k=\{1,2\}$ correspond to the degree of the matching functions.

improvement of spatial resolution may be obtained at the price of increasing sensitivity to noise, and vice versa. Moreover, this figure shows the power law relation between measurement and spatial resolution. An interesting property is that the slopes of the curves are the same, for both DIC versions and both kinematics degrees.

The metrological efficiency indicator as defined by Equation (1) combines both the spatial and the measurement resolutions to form one indicator only. The hypothesis is that this indicator is independent from the chosen element/subset size. Figure 7b shows this indicator for both the Local and the Global DIC versions, for linear and quadratic matching functions. They allow for a few observations:

- First, the indicator is nearly constant for larger element/subset sizes but deviates for small element/subset sizes. This is expected as the spatial resolution of the underlying speckle pattern will come into play for small elements/subsets, thereby increasing the indicator.

- Second, the difference between Local and Global DIC is small. This result explains the contradictory results found in the literature [23, 24] when approaching the problem more mathematically. When both methods are correctly applied, their performance is similar.

- Quadratic matching functions drastically improve the metrological efficiency indicator over linear ones. 


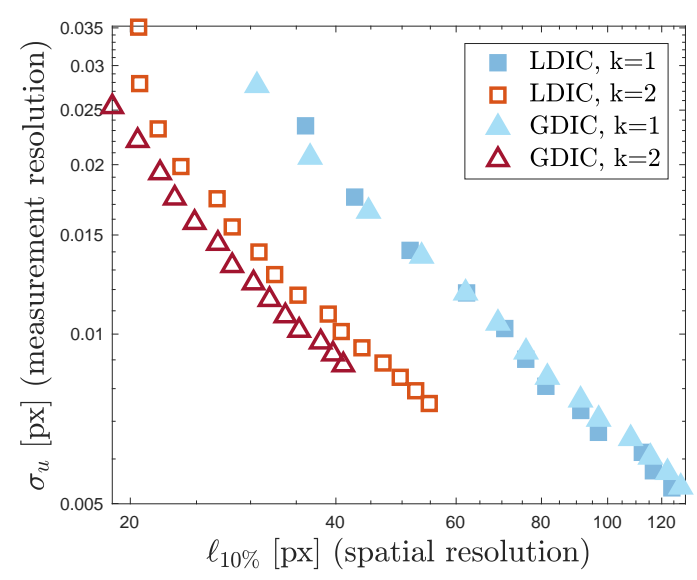

(a)

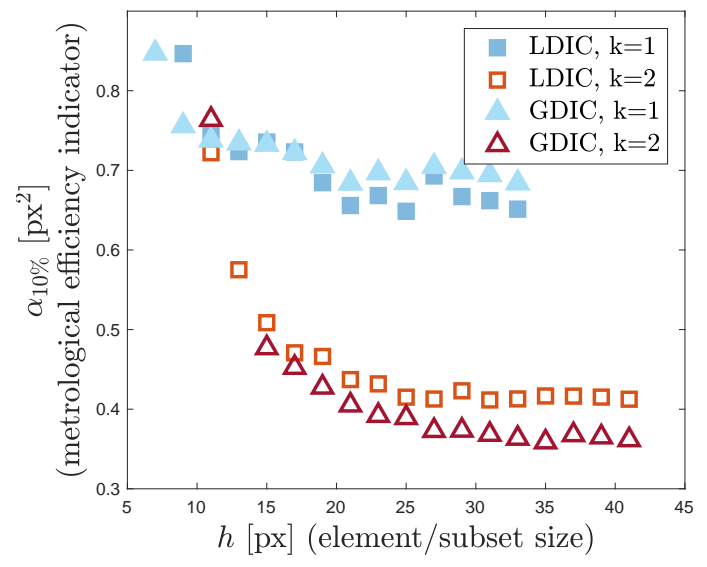

(b)

Figure 7: Metrological performance of Global and Local DIC: (a) measurement resolution as a function of the spatial resolution; (b) metrological efficiency indicator as a function of the support width, i.e. the element or the subset size. LDIC and GDIC respectively refer to the Local and Global version of DIC, and $k=\{1,2\}$ correspond to the degree of the matching functions. Local and Global DIC feature the same performance. Higher degree kinematics are characterized by a lower indicator, which means that they exhibit a better compromise between measurement and spatial resolutions. 


\subsection{Influence of the bias $\lambda$}

It is worth noting that the results and conclusions presented above only rely on calculations performed assuming a $10 \%$ bias. Since this choice impacts the spatial resolution, and thus the position of the border between Zones B and C in Figure 4a, it is of interest to study how it impacts the metrological efficiency indicator. This is presented in Figure 8, for biases ranging from $5 \%$ to $50 \%$. For a low bias, for instance below $10 \%$, the border between Zones B and C is located on the right-hand side in Figure 4a. This zone features low frequency displacements. Consequently, each of the DIC versions are applied in their region of confidence. The measured displacement will thus be close to the ground truth. In this case, Global DIC features a better metrological performance. This observation is emphasized when considering the second degree matching functions. The higher values reached by Local DIC are explained by its low pass filter behavior. On the contrary, for higher biases, above 30\% for instance, the border between Zones B and C is in a more challenging region, namely the left-hand side of Figure 4a. In that case, the low pass filtering effect of Local DIC enhances the measurement robustness. Interestingly, the degree of the matching functions has less impact in this case. This can be observed in Figure 8, where the metrological efficiency indicators of both first and second degrees matching functions approach each other for Local DIC. These results also explain the opposing conclusions about the metrological performance of Local and Global DIC found in the literature when approaching the problem for a fixed bias. In other words, the presented results only represent the two specific versions of the Local and Global implementations used in this article, and are not generalizable to any DIC method. The crossing points, where one method starts outperforming the other can easily shift depending on specific implementations.

\section{Conclusions}

This paper first recalled a versatile method for the characterization of the metrological performance of full-field measurement techniques. The measurement resolution corresponds to the random error, the bias to the systematic error and the spatial resolution reflects the cut-off wavelength, when considering full-field measurement techniques as a lowpass filtering method. The fact that this method does not rely on extrinsic parameters, which are technique- or user-dependent, enables fair comparisons between different measurement techniques. The method relies on synthetic images. Other experimental sources of error are thus not taken into account. They are however expected to impact all the full-field measurement techniques studied here in the same manner.

Thanks to this methodology, the assessment of the metrological performance of two specific versions of DIC, namely Local and Global DIC, has been carried out. For both methods an optimal setting is found in order to compare them. For Local DIC, this resulted in using a "dense" formulation by using a DIC calculation for every pixel, thus 


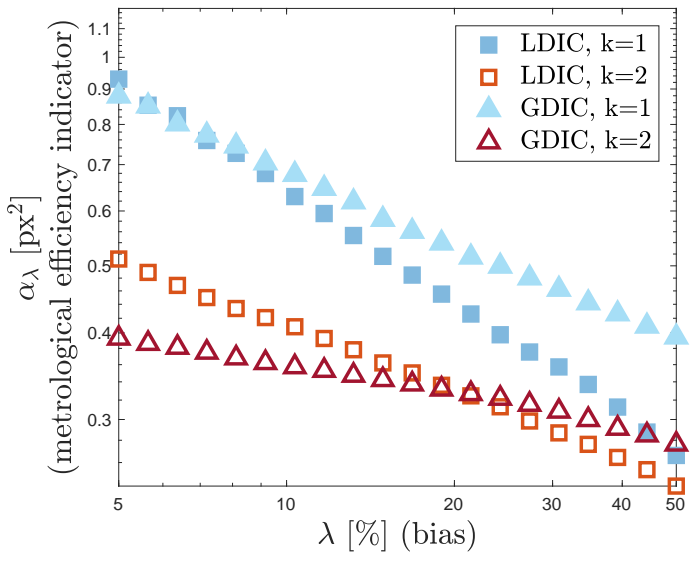

Figure 8: Metrological efficiency indicator as a function of the bias used for the spatial resolution calculation. LDIC and GDIC respectively refer to the Local and Global version of DIC and $k=\{1,2\}$ corresponds to the degree of the matching functions. The lower the metrology efficiency indicator, the better the metrological performances.

avoiding extra interpolation between the subsets. For Global DIC, all meshes were made of equilateral triangles, creating hexagonal grids. However, the location of the grid lines with respect to the underlying kinematics matters. Therefore, three different configurations were analyzed and the one that behaves in a similar manner to Local DIC was used.

A first conclusion is that both methods feature metrological efficiency indicators of the same order of magnitude, which means that they exhibit similar metrological performance. An interesting point is the equivalence between the spatial resolution for subsets/elements with the same edge length. Although the triangles used in the Global DIC implementation have a significantly smaller area, their respective connectivity to neighboring elements increases their support size. It becomes equivalent to the square subsets with the same edge length.

Given the similarity of both techniques, the relative performance can switch depending on the choice of the bias $\lambda$. Every choice of a kinematic description has a range of spatial frequencies, where it works well, which naturally has its limits beyond which it can not correctly represent the sought displacement. One way of looking at $\lambda$ is to consider how far the kinematic solution is allowed to push this limit. For very small values of $\lambda$, it corresponds to displacement frequencies that are far from the limits of the methods. Conversely, for larger values of $\lambda$, say $\lambda=20 \%$, it corresponds to displacement frequencies that can only be poorly represented by the kinematics. The proposed metrological efficiency indicator $\alpha_{\lambda}$ can be used to study the relative performance of the two methods as a function of the chosen bias. It was shown in this article that for larger biases (i.e. $\lambda>30 \%$ ) Local 
DIC method outperforms Global method. In practice, this comes from a filtering effect within the Local method that allows the method to give more conservative results when the underlying wavelength becomes too challenging to be described for the chosen subset size. Conversely, the absence of this filtering effect in the Global method allows it to both under- and over-estimate the curvature in the real kinematics. This becomes unstable in cases for which the element size is too large for the underlying kinematics, but this is an advantage for stricter choices for the bias. Consequently, it outperforms the Local method for small values of the bias (i.e. $\lambda<10 \%$ ).

Finally, this study confirms that DIC users should always implement second-degree DIC versions, to take benefit of the associated outstanding gain in terms of metrological performance.

\section{Acknowledgement}

The authors are grateful to the French National Research Agency (ANR) for their financial support (ICAReS project, NANR-18-CE08-0028-01).

\section{References}

[1] B. D. Lucas and T. Kanade. "An Iterative Image Registration Technique with an Application to Stereo Vision". In: Proceedings of the 7th International Joint Conference on Artificial Intelligence - Volume 2. IJCAI'81. San Francisco, CA, USA: Morgan Kaufmann Publishers Inc., 1981, pp. 674-679.

[2] M. Sutton, W. Wolters, W. Peters, W. Ranson, and S. McNeill. "Determination of displacements using an improved digital correlation method". In: Image and Vision Computing 1.3 (1983), pp. 133-139.

[3] M. A. Sutton, C. Mingqi, W. H. Peters, Y. J. Chao, and S. R. McNeill. "Application of an optimized digital correlation method to planar deformation analysis". In: Image and Vision Computing 4.3 (1986), pp. 143-150.

[4] B. Blaysat, M. Grédiac, and F. Sur. "Effect of interpolation on noise propagation from images to DIC displacement maps". In: International Journal for Numerical Methods in Engineering 108.3 (2016), pp. 213-232.

[5] B. Blaysat, M. Grédiac, and F. Sur. "On the propagation of camera sensor noise to displacement maps obtained by DIC - an experimental study". In: Experimental Mechanics 56.6 (2016), pp. 919-944.

[6] A. Baldi and F. Bertolino. "Experimental Analysis of the Errors due to Polynomial Interpolation in Digital Image Correlation". In: Strain (2015), pp. 248-263. 
[7] X. D. Ke, H. W. Schreier, M. A. Sutton, and Y. Q. Wang. "On Error Assessment in Stereo-based Deformation Measurements". In: Experimental Mechanics 51.4 (2011), pp. 423-441.

[8] Y. Q. Wang, M. A. Sutton, H. A. Bruck, and H. W. Schreier. "Quantitative Error Assessment in Pattern Matching: Effects of Intensity Pattern Noise, Interpolation, Strain and Image Contrast on Motion Measurements". In: Strain 45.2 (2009), pp. $160-178$.

[9] Y. Q. Wang, M. A. Sutton, X. D. Ke, H. W. Schreier, P. L. Reu, and T. J. Miller. "On Error Assessment in Stereo-based Deformation Measurements". In: Experimental Mechanics 51.4 (2011), pp. 405-422.

[10] H. W. Schreier and M. A. Sutton. "Systematic errors in digital image correlation due to undermatched subset shape functions". In: Experimental Mechanics 42.3 (2002), pp. 303-310.

[11] M. A. Sutton, S. R. McNeill, J. Jang, and M. Babai. "Effects Of Subpixel Image Restoration On Digital Correlation Error Estimates". In: Optical Engineering 27.10 (1988), pp. 870-877.

[12] B. Wang and B. Pan. "Random Errors in Digital Image Correlation Due to Matched or Overmatched Shape Functions". In: Experimental Mechanics 55.9 (2015), pp. 17171727.

[13] B. Pan. "Bias error reduction of digital image correlation using Gaussian pre-filtering". In: Optics and Lasers in Engineering 51.10 (2013), pp. 1161-1167.

[14] B. Pan, L. Yu, D. Wu, and L. Tang. "Systematic errors in two-dimensional digital image correlation due to lens distortion". In: Optics and Lasers in Engineering 51.2 (Feb. 2013), pp. 140-147.

[15] X. Xu, Y. Su, and Q. Zhang. "Theoretical estimation of systematic errors in local deformation measurements using digital image correlation". In: Optics and Lasers in Engineering 88 (Jan. 2017), pp. 265-279.

[16] M. Grédiac and F. Sur. "Effect of Sensor Noise on the Resolution and Spatial Resolution of Displacement and Strain Maps Estimated with the Grid Method". In: Strain 50.1 (2014), pp. 1-27.

[17] M. Grédiac, B. Blaysat, and F. Sur. "A Critical Comparison of Some Metrological Parameters Characterizing Local Digital Image Correlation and Grid Method". In: Experimental Mechanics 57.6 (2017), pp. 871-903.

[18] S. Roux, F. Hild, and Y. Berthaud. "Correlation Image Velocimetry: A Spectral Approach". In: Appl. Opt. 41.1 (2002), pp. 108-115.

[19] Y. Sun, J. H. L. Pang, C. K. Wong, and F. Su. "Finite element formulation for a digital image correlation method". In: Appl. Opt. 44.34 (Dec. 2005), pp. 7357-7363. 
[20] G. Besnard, F. Hild, and S. Roux. "Finite-element displacement fields analysis from digital images: application to Portevin-Le Châtelier bands." In: Experimental Mechanics 46.6 (2006), pp. 789-803.

[21] F. Mathieu, H. Leclerc, F. Hild, and S. Roux. "Estimation of Elastoplastic Parameters via Weighted FEMU and Integrated-DIC". In: Experimental Mechanics 55.1 (2015), pp. 105-119.

[22] A. P. Ruybalid, J. P. M. Hoefnagels, O. van der Sluis, and M. G. D. Geers. "Comparison of the identification performance of conventional FEM updating and integrated DIC". In: International Journal for Numerical Methods in Engineering 106.4 (2016), pp. 298-320.

[23] F. Hild and S. Roux. "Comparison of Local and Global Approaches to Digital Image Correlation". In: Experimental Mechanics 52 (2012), pp. 1503-1519.

[24] B. Pan, B. Wang, G. Lubineau, and A. Moussawi. "Comparison of Subset-Based Local and Finite Element-Based Global Digital Image Correlation". In: Experimental Mechanics 55.5 (2015), pp. 887-901.

[25] L. Wittevrongel, P. Lava, S. V. Lomov, and D. Debruyne. "A Self Adaptive Global Digital Image Correlation Algorithm". In: Experimental Mechanics 55.2 (2015), pp. 361378.

[26] M. Grédiac, F. Sur, and B. Blaysat. "The Grid Method for In-plane Displacement and Strain Measurement: A Review and Analysis". In: Strain 52.3 (2016), pp. 205243.

[27] E. A. Patterson, E. Hack, P. Brailly, R. L. Burguete, Q. Saleeme, T. Siebert, R. A. Tomlinsone, and M. P. Whelan. "Calibration and evaluation of optical systems for full-field strain measurement". In: Optics and Lasers in Engineering 45.5 (2007), pp. 550-564.

[28] E. Hack, G. Lampeas, J. Mottershead, E. Patterson, T. Siebert, and M. Whelan. "Progress in developing a standard for dynamic strain analysis". In: Experimental and Applied Mechanics. Vol. 6. Conference Proceedings of the Society for Experimental Mechanics Series. 2011, pp. 425-429.

[29] C. Sebastian, X. Lin, E. Hack, and E. Patterson. "A Reference Material for Establishing Uncertainty for Static and Dynamic Displacements". In: Proceedings of the SEM conference, Costa Mesa, CA, USA. 2015.

[30] E. Hack, X. Lin, E. Patterson, and C. Sebastian. "A reference material for establishing uncertainties in full-field displacement measurements". In: Measurement Science and Technology 26.7 (2015), p. 075004.

[31] http://cordis.europa.eu/result/brief/rcn/3262_en.html.

[32] http://www.dynamicvalidation.org/. 
[33] http://www.engineeringvalidation.org/.

[34] Standard guide for evaluating non-contacting optical strain measurement systems, ASTM standard E2208-02, 2010.

[35] ASD-STAN. prEN 4861 P1, Aerospace series-Metrological assessment procedure for kinematic fields measured by digital image correlation. Tech. rep. 2018, p. 36.

[36] M. A. Sutton, J. J. Orteu, and H. Schreier. Image correlation for shape, motion and deformation measurements: Basic Concepts, Theory and Applications. Springer, 2009 .

[37] W. Tong. "Reduction of Noise-Induced Bias in Displacement Estimation by Linear Off-Pixel Digital Image Correlation". In: Strain 49.2 (2013), pp. 158-166.

[38] Z. Y. Wang, H. Q. Li, J. W. Tong, and J. T. Ruan. "Statistical Analysis of the Effect of Intensity Pattern Noise on the Displacement Measurement Precision of Digital Image Correlation Using Self-correlated Images". In: Experimental Mechanics 47.5 (2007), pp. 701-707.

[39] J. Réthoré, G. Besnard, G. Vivier, F. Hild, and S. Roux. "Experimental investigation of localized phenomena using digital image correlation". In: Philosophical Magazine 88.28-29 (2008), pp. 3339-3355.

[40] J. Réthoré, F. Hild, and S. Roux. "Extended digital image correlation with crack shape optimization". In: International Journal for Numerical Methods in Engineering 73.2 (2008), pp. 248-272.

[41] S. Roux and F. Hild. "Stress intensity factor measurements from digital image correlation: post-processing and integrated approaches". In: International Journal of Fracture 140 (2006), pp. 141-157.

[42] M. Grédiac, F. Sur, C. Badulescu, and J.-D. Mathias. "Using deconvolution to improve the metrological performance of the grid method". In: Optics and Lasers in Engineering 51.6 (2013), pp. 716-734.

[43] F. Sur, B. Blaysat, and M. Grédiac. "Rendering Deformed Speckle Images with a Boolean Model". In: Journal of Mathematical Imaging and Vision 60.5 (2018), pp. 634-650.

[44] SEM - DIC Challenge. http://sem.org/content.asp?contentid=196.

[45] P. Reu, B. Blaysat, E. Jones, and J. Helm. "Update on the DIC Challenge 2.0 and the Stereo-DIC Challenge". In: SEM Conference, Reno, USA. 2019.

[46] A. Foi, M. Trimeche, V. Katkovnik, and K. Egiazarian. "Practical Poissonian-Gaussian Noise Modeling and Fitting for Single-Image Raw-Data". In: IEEE Transactions on Image Processing 17.10 (2008), pp. 1737-1754.

[47] B. Pan, H. Xie, and Z. Wang. "Equivalence of digital image correlation criteria for pattern matching". In: Appl. Opt. 49.28 (Oct. 2010), pp. 5501-5509. 
[48] W. Tong. "Formulation of Lucas-Kanade Digital Image Correlation Algorithms for Non-contact Deformation Measurements: A Review". In: Strain 49.4 (2013), pp. 313334 .

[49] B. Pan and B. Wang. "Digital Image Correlation with Enhanced Accuracy and Efficiency: A Comparison of Two Subpixel Registration Algorithms". In: Experimental Mechanics 56.8 (2016), pp. 1395-1409.

[50] J. Neggers, B. Blaysat, J. P. M. Hoefnagels, and M. G. D. Geers. "On image gradients in digital image correlation". In: International Journal for Numerical Methods in Engineering 105.4 (2016), pp. 243-260.

[51] J.-C. Passieux and R. Bouclier. "Classic and Inverse Compositional Gauss-Newton in Global DIC". In: International Journal for Numerical Methods in Engineering (2019), In Press.

[52] J. Huang, X. Pan, X. Peng, Y. Yuan, C. Xiong, J. Fang, and F. Yuan. "Digital Image Correlation with Self-Adaptive Gaussian Windows". In: Experimental Mechanics 53.3 (2013), pp. 505-512.

[53] V. Valle, S. Hedan, P. Cosenza, A. L. Fauchille, and M. Berdjane. "Digital Image Correlation Development for the Study of Materials Including Multiple Crossing Cracks". In: Experimental Mechanics 55.2 (2015), pp. 379-391.

[54] M. Grédiac, B. Blaysat, and F. Sur. "A robust-to-noise deconvolution algorithm to enhance displacement and strain maps obtained with local DIC and LSA". In: Experimental Mechanics 59.2 (2019), pp. 219-243.

[55] R. Fedele, L. Galantucci, and A. Ciani. "Global 2D digital image correlation for motion estimation in a finite element framework: a variational formulation and a regularized, pyramidal, multi-grid implementation". In: International Journal for Numerical Methods in Engineering 96.12 (2013), pp. 739-762.

[56] J. Neggers, J. P. M. Hoefnagels, F. Hild, S. Roux, and M. G. D. Geers. "A Global Digital Image Correlation Enhanced Full-Field Bulge Test Method". In: Procedia IUTAM 4.0 (2012), pp. 73-81.

[57] J.-E. Dufour, F. Hild, and S. Roux. "Integrated digital image correlation for the evaluation and correction of optical distortions". In: Optics and Lasers in Engineering 56.0 (2014), pp. 121-133.

[58] S. M. Kleinendorst, J. P. M. Hoefnagels, C. V. Verhoosel, and A. P. Ruybalid. "On the use of adaptive refinement in isogeometric digitalimage correlation". In: International Journal for Numerical Methods in Engineering 104.10 (2015), pp. 944-962.

[59] J. Rupil, S. Roux, F. Hild, and L. Vincent. "Fatigue microcrack detection with digital image correlation". In: The Journal of Strain Analysis for Engineering Design 46.6 (2011), pp. 492-509. 
[60] F. Mathieu, F. Hild, and S. Roux. "Identification of a crack propagation law by digital image correlation". In: International Journal of Fatigue 36.1 (2012), pp. 146-154.

[61] B. Blaysat, J. P. M. Hoefnagels, G. Lubineau, M. Alfano, and M. G. D. Geers. "Interface debonding characterization by image correlation integrated with Double Cantilever Beam kinematics". In: International Journal of Solids and Structures 55 (2015), pp. 79-91.

[62] J. Réthoré. "A fully integrated noise robust strategy for the identification of constitutive laws from digital images". In: International Journal for Numerical Methods in Engineering 84.6 (2010), pp. 631-660.

[63] R. Gras, H. Leclerc, S. Roux, S. Otin, J. Schneider, and J.-N. Périé. "Identification of the Out-of-Plane Shear Modulus of a 3D Woven Composite". In: Experimental Mechanics (2012), pp. 1-12.

[64] J.-C. Passieux, F. Bugarin, C. David, J.-N. Périé, and L. Robert. "Multiscale Displacement Field Measurement Using Digital Image Correlation: Application to the Identification of Elastic Properties". In: Experimental Mechanics 55.1 (2015), pp. 121137 .

[65] J. Neggers, J. P. M. Hoefnagels, M. G. D. Geers, F. Hild, and S. Roux. "Time-resolved integrated digital image correlation". In: International Journal for Numerical Methods in Engineering 103.3 (2015), pp. 157-182.

[66] J. Neggers, J. P. M. Hoefnagels, F. Hild, S. Roux, and M. G. D. Geers. "Direct stressstrain measurements from bulged membranes using topography image correlation". In: Experimental Mechanics 54.5 (2014), pp. 717-727.

[67] A. Baldi and F. Bertolino. "Assessment of h-refinement procedure for global digital image correlation". In: Meccanica 51.4 (2016), pp. 979-991.

[68] F. Sur and M. Grédiac. "Influence of the Analysis Window on the Metrological Performance of the Grid Method". In: Journal of Mathematical Imaging and Vision 56.3 (2016), pp. 472-498.

[69] M. Grédiac, B. Blaysat, and F. Sur. "On the optimal pattern for displacement field measurement: random speckle and DIC, or checkerboard and LSA?" In: Experimental Mechanics (2020).

[70] JCGM Member Organizations. International vocabulary of metrology - Basic and general concepts and associated terms (VIM). Vol. 200. BIPM, 2012.

[71] A. Chrysochoos and Y. Surrel. "Chapter 1. Basics of metrology and introduction to techniques". In: Full-field measurements and identification in solid mechanics. Ed. by M. Grédiac and F. Hild. Wiley, 2012, pp. 1-29. 
[72] M. Bornert, F. Brémand, P. Doumalin, J. C. Dupré, M. Fazzini, M. Grédiac, F. Hild, S. Mistou, J. Molimard, J. J. Orteu, L. Robert, Y. Surrel, P. Vacher, and B. Wattrisse. "Assessment of Digital Image Correlation Measurement Errors: Methodology and Results". In: Experimental Mechanics 49 (2009), pp. 353-370.

[73] J. Blaber, B. Adair, and A. Antoniou. "Ncorr: Open-Source 2D Digital Image Correlation Matlab Software". In: 55.6 (2015), pp. 1105-1122.

\section{A Appendix: vocabulary and definitions}

Three metrological parameters are discussed in this paper, namely the measurement resolution, the bias and the spatial resolution. Their definition, already given in $[17,69]$, are recalled below for the sake of complitness:

- Measurement resolution: in Ref. [70], the measurement resolution is defined by the smallest change in a quantity being measured that causes a perceptible change in the corresponding indication. More precisely, it is proposed in [71] to define it as the change in quantity being measured that causes a change in the corresponding indication greater than one standard deviation of the measurement noise, which enables us to quantify the measurement resolution. This definition is quite arbitrary, any other (reasonable) multiple of the standard deviation being also potentially acceptable, but the idea is that the resolution quantifies the smallest change not likely to be caused by measurement noise [71].

- Spatial resolution: the spatial resolution denoted by $\ell_{\lambda}$ is defined here by the lowest period of a sinusoidal deformation that the technique is able to reproduce before losing a certain percentage $\lambda$ of amplitude, this quantity being chosen a priori [25]. The advantage of this definition is that it is not based on an arbitrary value for the subset size in Local DIC or for the elements size in Global DIC. This makes it possible to compare the spatial resolution between these two techniques.

- Bias: a systematic error generally occurs when a given technique returns actual details in displacement and strain maps. It is due to the fact that the amplitude of such apparent details is generally lower than the amplitude of the actual detail. This apparent "damping" is a bias, which can be quantified by considering a sinusoidal reference displacement field, and measuring the relative loss of amplitude exhibited by the displacement field returned by the technique under study, as suggested in Refs. [25, 44, 72, 73]. Of course, the loss of amplitude depends on the frequency $f$ of the sine function. This loss of amplitude is denoted here by $l(f)$. In this context, the spatial resolution defined above is defined for a given bias $\lambda$, the relation between $\ell_{\lambda}$ and $\lambda$ being that $\ell_{\lambda}$ is the smallest value such that $l\left(1 / \ell_{\lambda}\right)=\lambda$. We call here $\lambda$ the bias of the method. This is a slight abuse of language since fixing $\lambda$ does not mean 
that the damping of any displacement or strain field is actually equal to this $\lambda$ value. Note finally that for DIC, the effect quantified here by $\lambda$ is often referred to as the "matching bias", because it occurs when there is a mismatch between the matching function used to describe the displacement within subsets on the one hand, and the degree of the actual displacement if the latter is described by a polynomial on the other hand. 